\title{
A SIMPLE DERIVATION OF AND IMPROVEMENTS TO JAMSHIDIAN'S AND ROGERS' UPPER BOUND METHODS FOR BERMUDAN OPTIONS
}

\author{
MARK S. JOSHI
}

\begin{abstract}
The additive method for upper bounds for Bermudan options is rephrased in terms of buyer's and seller's prices. It is shown how to deduce Jamshidian's upper bound result in a simple fashion from the additive method, including the case of possibly zero final pay-off. Both methods are improved by ruling out exercise at sub-optimal points. It is also shown that it is possible to use sub- Monte Carlo simulations to estimate the value of the hedging portfolio at intermediate points in the Jamshidian method without jeopardizing its status as upper bound.
\end{abstract}

\section{INTRODUCTION}

The pricing of options involving early exercise opportunities by Monte Carlo is a classic hard problem. Most of the work on the topic has been focussed on developing unbiased estimates of lower bounds. However, one can never be sure of the efficacy of lower bounds without a matching upper bound. If the bounds are close together, one can be sure that both are good. For a general survey of the area, we refer the reader to [4].

Two upper bounds methods have recently become popular: those of Rogers, [11], and Jamshidian, [7], and extensions thereof. Haugh and Kogan developed a method similar to Rogers at around the same time, [6], as our approach is more in line with Rogers, we shall talk here of Rogers' method. The Rogers/Haugh and Kogan method is sometimes called the additive method, whilst Jamshidian's method is called the multiplicative method. Both of these methods involve rephrasing the Bermudan option pricing problem in terms of a minimization problem using a hedging portfolio. We will show that they can be reinterpreted in terms of seller's prices and that this gives simpler derivations of their principal formulas.

Date: January 13, 2006.

Key words and phrases. Monte Carlo simulation, Bermudan option, American option. 
The methods differ in two ways: the precise choice of hedging portfolio, and in evaluating the maximum of a ratio instead of the maximum of a difference.

In fact, the precise choice of hedge is not important; in Rogers' method excess cash is used to buy numeraire bonds, whereas in Jamshidian's it is used to purchase extra units of the original hedge. We will see that if these strategies are swapped both methods are still correct.

An issue with Jamshidian's method is that the method relies on using the hedge as numeraire, and that the hedge can be of zero value at the final time frame. We show how this problem can be avoided by replacing the final exercise value by the price of a European option a small time $\delta$ before and then letting $\delta$ tend to 0 . This is an alternative to the argument in [7] which relies on adding $\epsilon$ zero-coupon bonds to the numeraire and letting $\epsilon \rightarrow 0$.

Both methods involve taking a maximum along each path which can be thought of a look-back option or exercise with maximal foresight. A curiosity is that the maximum of the hedged portfolio could occur at a point which is out-of-the-money or suboptimal to exercise. We show that such points can be excluded. (A similar more restricted result for the Rogers' method has also been found independently by Broadie and Cao, [2].)

An effective way to implement Rogers' method is to pick an exercise strategy, and then use the derivative with this exercise strategy and a reinvestment rule as the hedge. The estimation of the value of the hedge then requires Monte Carlo simulations and it is necessary to show that the Monte Carlo errors only cause upwards bias. This approach was explored by Anderson and Broadie in [1]. We show that for the strategy of reinvesting all additional cash in the original hedge, this approach also applies for the Jamshidian method.

We fix notation. Throughout, we will have a Bermudan option, $D$, which is exercisable on a discrete set of dates, $0<t_{1}<t_{2}<\cdots<t_{n}$. The pay-off on date $t_{j}$ is $Z_{j}$. We assume the possibility of non-exercise and therefore take $Z_{n} \geq 0$. We do not make this assumption at previous times. The value of the numeraire at time $t_{j}$ will be denoted by $N_{j}$. We denote expectation under the pricing measure associated with $N$ by $\mathbb{E}_{N}$. We will use $H_{j}$ to denote the value of a hedging portfolio at time $t_{j}$. Following Jamshidian, we restrict attention to Bermudan options since these are the only options which are practical to price in a computer. We also assume that we are working in a complete market throughout (or an incomplete market to which sufficient hedging instruments have been added to make the pricing measure for each numeraire unique.) 
We do not discuss here which of the two methods in better. This question has been examined, however, by Chen and Glasserman, [3], and by van der Helm, [5]. The results seem inconclusive with both methods sometimes better. The main virtue of the Rogers' method is, however, lower variance.

The structure of this paper is as follows. In Section 2, we review Rogers' result and rephrase it in terms of buyer's and seller's prices. In Section 3, we show how the Jamshidian method for positive final pay-offs can be deduced from the Rogers method. We address the issue of possibly zero final pay-offs in Section 4. Tweaks to the two methods which allow the avoidance of points of sub-optimal exercise are discussed in Section 5. We demonstrate in Section 6 that the use of sub-simulations does not cause downwards bias in the Jamshidian method. Our conclusion is in section 7 .

I am grateful to Farshid Jamshidian for comments on an earlier version of this paper.

\section{Rogers' RESULT}

We rederive Rogers' result in terms of buyer's and seller's prices. We also show that Rogers' result holds for Jamshidian's hedging portfolio. We will then use Rogers' result to deduce Jamshidian's result. The value of a Bermudan option is typically written as

$$
D_{0}=N_{0} \sup _{\tau} \mathbb{E}_{N}\left(Z_{\tau} N_{\tau}^{-1}\right)
$$

where the supremum is taken over all stopping times. In fact, this supremum has a natural maximum element which is to exercise then the exercise value is greater than or equal to the continuation value. This reflects the buyer's price for the option in that the buyer chooses the exercise strategy and this is then the amount of value he can realize.

The seller on the other hand cannot choose an exercise strategy but must hedge against the possibility of the buyer choosing any exercise strategy. Indeed if the buyer's chooses an exercise date at random then there is a non-zero probability of exercising at the maximum along the path. The seller must therefore be able to hedge against a buyer who can exercise at the maximum. This sounds hard but if we think in terms of a trader managing a book, it is clear that this can be done. The seller hedges under the assumption that the buyer is following the optimal strategy, and so "buys" one unit of the Bermudan option to hedge with for the buyer's price. At each exercise date, one of four things can happen. In the two cases, where the buyer and seller agree then there is perfect hedging. If the buyer exercises and the seller does not then 
the price for the derivative from the optimal strategy is greater than the exercise value and the seller makes extra money and is more than hedged. If the buyer does not exercise and the seller does, then the seller can buy the unexercised option for less than the exercise value and is ahead again. The extra cash can be used to buy any instrument which is always of non-negative value. Rogers implicitly suggests numeraire bonds, whilst Jamshidian uses the money to buy more units of the original option. In fact, Jamshidian notes in [7] that there are many possible "domineering" hedges which will differ according to how this cash is reinvested.

Thus we have a constructed a hedging portfolio, $H_{t}$, such that $H_{0}$ equals $D_{0}$ and $H_{j} \geq Z_{j}$ for all $j$ along each path. We therefore have for any numeraire $N$

$$
N_{0} \mathbb{E}\left(\max _{j} \frac{Z_{j}-H_{j}}{N_{j}}\right)=0 .
$$

If we now restrict to hedges of initial value 0 , as Rogers does, we can subtract units of the numeraire of initial value $D_{0}$ to obtain,

$$
\begin{aligned}
D_{0} & =H_{0}+N_{0} \mathbb{E}_{N}\left(\max _{j} \frac{Z_{j}-H_{j}}{N_{j}}\right), \\
& =N_{0} \mathbb{E}_{N}\left(\max _{j} \frac{Z_{j}-\left(H_{j}-H_{0} N_{j}\right)}{N_{j}}\right),
\end{aligned}
$$

where, of course, $D_{0}=H_{0}$. We have shown that the option can be hedged even if the buyer acts with maximal foresight. This shows that buyer's and seller's prices are equal. This point of view is mentioned in [10].

Rogers' method is in two parts, the first is that inequality holds in (2.4) for any $H$ of initial value zero, and the second is that equality holds for the correct hedge which we have already shown. To see that the inequality holds for any $H$ of initial value zero, we observe that

$$
0=H_{0}=N_{0} \sup _{\tau} \mathbb{E}_{N}\left(H_{\tau} N_{\tau}^{-1}\right),
$$

by the Optional Sampling theorem, since we are sampling a martingale at a bounded stopping time. (see e.g. [9].) We therefore have

$$
D_{0}=N_{0} \sup _{\tau} \mathbb{E}_{N}\left(\left(Z_{\tau}-H_{\tau}\right) N_{\tau}^{-1}\right) \text {. }
$$

If we take the supremum over the set of all random times instead of over all stopping times, the value can only increase and there is a clear best choice: the maximum along the path. So we have

$$
D_{0} \leq N_{0} \mathbb{E}_{N}\left(\max _{j}\left(Z_{j}-H_{j}\right) N_{j}^{-1}\right) \text {. }
$$




\section{REDERIVING JAMSHIDIAN'S RESUlT FOR POSITIVE PAY-OFFS}

We first suppose that $Z_{n}>0$ and restrict ourselves to hedges, $H_{t}$, which are always of positive value. We can therefore take the hedge to be numeraire. We have from above,

$$
\begin{aligned}
D_{0} & =H_{0}+H_{0} \mathbb{E}_{H}\left(\max _{j} \frac{Z_{j}-H_{j}}{H_{j}}\right), \\
& =H_{0} \mathbb{E}_{H}\left(\frac{H_{j}}{H_{j}}+\max _{j} \frac{Z_{j}-H_{j}}{H_{j}}\right), \\
& =H_{0} \mathbb{E}_{H}\left(\max _{j} \frac{Z_{j}}{H_{j}}\right) .
\end{aligned}
$$

We can identify this as the price of a look-back option paying

$$
\max _{j} \frac{Z_{j}}{H_{j}}
$$

units of $H_{n}$ at time $t_{n}$. Applying change of numeraire, for an arbitrary numeraire $N$ we have

$$
D_{0}=N_{0} \mathbb{E}_{N}\left(\frac{H_{n}}{N_{n}} \max _{j} \frac{Z_{j}}{H_{j}}\right) .
$$

For the optimal hedge, we therefore have equality. For another hedge of positive value everywhere, it is sufficient to observe that the entire derivation above is still valid provided we replace "=" by " $\leq$ " everywhere, and so we have

$$
D_{0} \leq N_{0} \mathbb{E}_{N}\left(\frac{H_{n}}{N_{n}} \max _{j} \frac{Z_{j}}{H_{j}}\right) .
$$

\section{REDERIVING JAMSHIDIAN'S RESULT FOR NON-NEGATIVE PAY-OFFS}

Nearly all financial examples have the possibility that $Z_{n}$ is equal to zero. For example, a call or put option may finish out of the money. One solution is to stop at time $t_{n-1}$ replacing the pay-off at that time by the maximum of the European value for $t_{n}$ and the pay-off at that

time. This, however, has the disadvantage that the pay-off becomes highly model dependent and relies on the easy availability of an analytic formula for the European price. It is therefore desirable to have an approach that is less model dependent.

One can manipulate expression (3.5), to read

$$
D_{0} \leq N_{0} \mathbb{E}_{N}\left(\max \left(\frac{Z_{n}}{N_{n}}, \frac{H_{n}}{N_{n}} \max _{j<n} \frac{Z_{j}}{H_{j}}\right)\right),
$$


and similarly with equality for the optimal hedge. This equation makes sense even when $H_{n}$ can be zero with non-zero probability. It remains to justify it, however.

We assume that the European option expiring at time $t_{n}$ with payoff $Z_{n}$ is of positive value with probability 1 before time $t_{n}$. This is a condition that Jamshidian calls "semi-positivity." If $Z_{n}$ is a function purely of the market state at time $t_{n}$, that is it not path-dependent, then the condition essentially states that the set $Z_{n}>0$ can always be reached with positive probability. This will hold for a large variety of models including Black-Scholes, jump-diffusion, stochastic volatility, and CGMY. Note it does not hold for a CEV model where the probability of absorption at zero is greater than zero.

Define $D^{\delta}$ for $\delta<t_{n}-t_{n-1}$ to be the original Bermudan option with the final exercise time replaced by $t_{n}-\delta$ and the final pay-off replaced by the model price of the European option on $Z_{n}$ expiring at time $t_{n}$. The price of $D^{\delta}$ will agree with that of $D$ since after time $t_{n-1}$, the value of $D$ is that of the European option expiring at time $t_{n}$.

Our assumption on the model and pay-off implies that the final payoff of $D^{\delta}$ is almost surely positive. So if we let $H^{\delta}$ equal the optimal hedging portfolio associated to the $D^{\delta}$ option then the results of the previous section hold. Letting $N_{n}^{\delta}$ be the value of $N$ at time $t_{n-\delta}$.

We therefore have

$$
D_{0}=N_{0} \mathbb{E}_{N}\left(\max \left(\frac{Z_{n}^{\delta}}{N_{n}^{\delta}}, \frac{H_{n}^{\delta}}{N_{n}^{\delta}} \max _{j<n} \frac{Z_{j}}{H_{j}}\right)\right),
$$

with $H$ the optimal hedge. Note that except at the final time frame $H^{\delta}$ and $H$ will agree. Letting $\delta$ tend to zero, the value of $H_{n}^{\delta}$ converges to that of $H_{n}$, and similarly for $N$. We obtain

$$
D_{0}=N_{0} \mathbb{E}_{N}\left(\max \left(\frac{Z_{n}}{N_{n}}, \frac{H_{n}}{N_{n}} \max _{j<n} \frac{Z_{j}}{H_{j}}\right)\right),
$$

as required.

For sub-optimal hedges, a similar derivation with inequalities holds as long as the value of the hedge before final expiry is always strictly positive, and we have that (4.1) still holds.

\section{TWEAKING THE METHODS}

A curiosity in both Rogers' method and Jamshidian's method is that the maximum is taken over a collection of points where $Z_{j}$ could be zero. At such a point the buyer would never exercise and so we are taking a maximum over unnecessary points. In Rogers' method, this

could increase the maximum as we can considering the maximum of 
$Z_{j}-H_{j}$ which could be bigger than zero at such a point. As well as points out of the money, we can also consider that the buyer would never exercise at points that are provably sub-optimal. An example is a point in the money where the exercise value is less than the maximal European value.

Suppose we redefine $Z_{j}$ for $j<n$ to be equal to minus infinity at all points that are provably sub-optimal. We do not change the value at time $t_{n}$ to take account of the possibility of non-exercise. The buyer's price will not change since the optimal exercise strategy will not exercise at a such point in any case. However, our maxima along paths in both of the Rogers and Jamshidian methods should now be taken over a restricted set, as clearly the maximum will not be minus infinity. Denoting provably suboptimal exercise times with $j<n$ by SubOpt, we have

$$
D_{0} \leq N_{0} \mathbb{E}_{N}\left(\max \left(\frac{Z_{n}}{N_{n}}, \frac{H_{n}}{N_{n}} \max _{j<n, j \notin \mathrm{SubOpt}} \frac{Z_{j}}{H_{j}}\right)\right),
$$

and

$$
D_{0} \leq N_{0} \mathbb{E}_{N}\left(\max _{j \notin \text { Subopt }}\left(Z_{j}-H_{j}\right) N_{j}^{-1}\right) .
$$

Note independently Broadie and Cao, [2], proved using Snell envelope arguments the restricted result that one can exclude out-of-the-money points in Rogers' method before the first in-the-money point.

The ability to use a restricted set of points is particularly useful when instead of using a hedge given by an analytic formula one estimates the hedge value by Monte Carlo as in [1]. The number of sub-simulations is then greatly reduced allowing the possibility of substantial speed ups - the simulations will be particularly fast as close to the exercise boundary they will terminate much faster than out of the money.

\section{USING SUB-SIMULATIONS WITH JAMSHIDIAN'S METHOD}

This raises the question of whether the Anderson-Broadie approach of using an approximate hedge found by using an approximate stopping rule can be applied to the Jamshidian case. In this case, an exercise strategy is fixed and the hedge is the Bermudan option with that strategy. If the strategy is close to optimal then the hedge should also be close to optimal. The subtlety arises in that the value of the hedging portfolio must be estimated via Monte Carlo simulation rather than evaluated precisely via an analytic expression. In [1], Anderson and Broadie showed that the additional errors only caused upwards bias in Rogers' method, and this meant that the estimated quantity was still an unbiased estimate of an upper bound. 
We now wish to prove the same result for Jamshidian's method. We want to estimate the quantity from (4.1). Each quantity $H_{j}$ will, however, be estimated by Monte Carlo simulation. Since we are rolling over excess cash, there will be two sources of errors, the error in the simulation at time $t_{j}$ and the accumulated errors along the path from previous repurchases. We are therefore simulating for each path:

$$
\max \left(\frac{Z_{n}}{N_{n}}, \max _{j<n} \frac{Z_{j}}{H_{j} A_{j}} \frac{A_{n} H_{n}}{N_{n}}\right),
$$

where $A_{j}$ is the ratio of the estimated value of the hedge to the true value. For any given path, the maximum will be realized at some point $k$ so either it occurs before $n$ and we have

$$
\max _{j<n} \frac{Z_{j}}{H_{j}} \frac{H_{n}}{N_{n}}=\frac{Z_{k}}{H_{k}} \frac{H_{n}}{N_{n}},
$$

or if $k=n$, then there is no error estimate at time slice $n$ and introducing the maximum across the other times can only increase values so we are done. Otherwise, it is enough to show that

$$
\mathbb{E}\left(\frac{Z_{k}}{H_{k} A_{k}} \frac{H_{n} A_{n}}{N_{n}}\right) \geq \frac{Z_{k}}{H_{k}} \frac{H_{n}}{N_{n}} .
$$

In other words, we need to show

$$
\mathbb{E}\left(\frac{A_{n}}{A_{k}}\right) \geq 1
$$

The term $A_{n}$ will be accumulated hedging errors along the path with no error in the pricing at time $t_{n}$ as that is the final pay-off. The term $A_{k}$ will be the product of rehedging errors up to time $t_{k}$ and if time $t_{k}$ is a point of non-exercise an additional pricing error, there too.

Let $B_{j}$ be the ratio of the Monte Carlo estimate of the price of one unit of the original unexercised Bermudan with the fixed exercise strategy at time $t_{j}$ to its true value. We have that variables $B_{j}$ are independent (assuming we use different seeds for the Monte Carlo simulations) positive and of mean 1.

Since $1 / x$ is a convex function for $x>0$, we also have from Jensen's inequality that

$$
\mathbb{E}\left(B_{j}^{-1}\right) \geq 1
$$

The term $A_{n} / A_{k}$ will be a product of independent, individual terms $B_{j}$ and $B_{j}^{-1}$, and will therefore have mean greater than or equal to 1 , and we are done.

Note that if one were to carry out this approach there would be additional difficulties. In particular, when deeply out of the money, it is possible that the estimate of the hedge value could be zero leading 
to infinite terms. This could be avoided either by importance sampling to ensure a sufficient number of paths were in the money or by the use of a control variate such as the maximal European option.

\section{Conclusion}

We have explored various aspects of Rogers' method and Jamshidian's method for upper bounds. We have shown how these are both theoretically related to the concept of a seller's price for a Bermudan option, and given easy derivations of their theoretical optimality. We have also seen how sub-optimal points can be removed from each method by redefining the pay-off to be minus infinity at such points. The use of a sub-simulation method for Jamshidian's approach has also been shown to be valid.

\section{REFERENCES}

[1] L. Anderson, M. Broadie, A primal-dual simulation algorithm for pricing multidimensional American options, Management Science, 2004, Vol. 50, No. 9, pp. 1222-1234.

[2] M. Broadie, M. Cao, Improving the efficiency of the primal-dual simulation algorithm for pricing American-style options. Working Paper, 2003, Columbia University, New York, USA.

[3] N. Chen, P. Glasserman, Additive and multiplicative duals for American option pricing, preprint Dec 2005.

[4] P. Glasserman, Monte Carlo Methods in Financial Engineering, Springer Verlag, 2003.

[5] W. van der Helm, Upper bound computation for Bermudan swaptions in the LIBOR market model, Bachelor's thesis Erasmus university

[6] M.Haugh, L. Kogan, Pricing American Options: A Duality Approach, Operations Research, 2004, 52:258-270

[7] F. Jamshidian, Numeraire-invariant option pricing and american, bermudan, and trigger stream rollover, Version 1.6, preprint July 2004

[8] M.S. Joshi, The concepts and practice mathematical finance, Cambridge University Press 2003

[9] I. Karatzas, E. Shreve, Brownian Motion and Stochastic Calculus, Second edition, Springer Verlag, 1997

[10] I. Karatzas, E. Shreve, Methods of Mathematical Finance, Springer Verlag, 1998

[11] L.C.G. Rogers: Monte Carlo valuation of American options, Mathematical Finance, Mathematical Finance, Vol. 12, pp. 271-286, 2002

Centre for Actuarial Studies, Department of Economics, UniverSity of Melbourne, Victoria 3010, Australia

E-mail address: mark@markjoshi.com 


\section{University Library}

\section{- M M I N E R VA A gateway to Melbourne's research publications}

Minerva Access is the Institutional Repository of The University of Melbourne

Author/s:

Joshi, Mark S.

Title:

A simple derivation of and improvements to Jamshidian's and Rogers' upper bound methods for Bermudan options

Date:

2006-03

Citation:

Joshi, Mark S. (2006) A simple derivation of and improvements to Jamshidian's and Rogers' upper bound methods for Bermudan options.

Persistent Link:

http://hdl.handle.net/11343/34297 